\title{
Galerkin Versions of Nonsingular Trefftz Method Derived from Variational Formulations for 2D Laplace Problem
}

\author{
ADAM BRAŃSKI $^{a, *}$ AND DOROTA BORKOWSKA ${ }^{b}$ \\ ${ }^{a}$ Laboratory of Acoustics, The Faculty of Electrical and Computer Engineering, Rzeszow University of Technology, \\ Powstańców Warszawy 12, 35-959 Rzeszow, Poland \\ ${ }^{b}$ Department of Computer Engineering in Management, The Faculty of Management, \\ Rzeszow University of Technology, Powstańców Warszawy 12, 35-959 Rzeszow, Poland
}

\begin{abstract}
In the paper, application of the Trefftz complete functions and Kupradze functions in two variational formulations is compared. They are applied in the original formulation and in the inverse one to the solution of boundary value problems of two-dimensional Laplace's equation. In these formulations, both solution and weighting functions are assumed to be of the same type, either the Trefftz function or the Kupradze function. Thus Galerkin versions of the methods are considered. All methods lead to the BEM and they are nonsingular. The relationship between the groups of methods of the original and inverse formulations is noticed. Numerical experiments are conducted for the Motz's problem. The accuracy and simplicity of the methods are discussed. All methods give comparable results. Since they are nonsingular, they may be successfully applied to solving boundary problems.
\end{abstract}

DOI: 10.12693/APhysPolA.128.A-50

PACS: 02.60.Lj, 02.30.Jr, 41.20.Cv

\section{Introduction}

In 1926, Trefftz [1] proposed a method of solving boundary problems for which the solution of homogeneous PDE exists in a close form. In point of fact, this is a boundary method which practically leads to the peculiar, non-singular boundary element method (BEM). Its application is the same as this of standard BEM, e.g. [24]. The Trefftz method (briefly, TM) employs superposition of functions (bases) satisfying homogeneous governing equation in a domain but not necessarily boundary conditions. The unknown coefficients are obtained from the criterion of meeting boundary conditions in any sense. In standard TM, the bases are Trefftz complete functions (T-functions) $[5,6]$. The general theory of TMs (groups of TM) can be found in [7]. The T-functions are named in different ways, e.g. the harmonic polynomials [8-10]. They are used in methods which are also named in different ways, for example the method of particular solutions [8].

Kupradze functions (briefly, K-functions) play quite the same role. They are in fact the fundamental solutions (FS) of the governing problem with the singularity removed by the application of the idea proposed by Kupradze, i.e. the source points are moved outside the domain $\Omega$ and the boundary $\Gamma$. So the K-functions satisfy homogeneous governing equation inside the domain of interest but not boundary conditions. It leads to the method which is named variously $[8,11]$. Some of the names that are quoted in [6] are: the modified Treffz method, the method of fundamental solutions, the source function method, or the discrete singularities method.

*corresponding author; e-mail: abranski@prz.edu.pl
Both of the above-mentioned methods belong to TMs. The comparison of these methods was first given in [12] and then in [8-10], and [13], but not in Galerkin versions. Therefore the aim of this paper is to compare these methods in this way. Other Trefftz-type methods are compared in $[11,14,15]$ where one of the two methods is applied in the Galerkin version.

In this work, four methods are derived. In order to distinguish the detailed differences among them, an auxiliary nomenclature is introduced in one of the following sections. The methods are derived from the original and the inverse variational formulations. The solutions are in the form of series in which the bases are either T-functions or K-functions. Since the Galerkin versions are considered, then the weights have the same forms as the bases, i.e. either T-functions or K-functions. All methods lead to the first kind of Fredholm BIE. The BIE is an inherent part of BEM; an excellent survey of BEM is given in [16].

First, the methods are formulated. Since the Laplace's equation is the simplest elliptic second order one, thus it is utilized for studying robustness and efficiency of the derived TMs. Numerical experiments are provided for the Motz's problem because this problem has the analytical solution. The results are depicted in figures and furthermore the errors of methods, via Euclidean norm, are given quantitatively. At the end, some conclusions are derived.

\section{Classical and variational formulations of boundary problems}

Let in the domain $\Omega$ surrounded by the boundary $\Gamma$ be given the Laplace boundary value problem described by a differential equation (Fig. 1)

$\Delta u(\boldsymbol{x})=0, \quad \boldsymbol{x}=\dot{\boldsymbol{x}} \in \Omega$, 


$$
\begin{aligned}
& u(\boldsymbol{x})=\widehat{u}(\boldsymbol{x}), \quad x \in \Gamma_{u} ; \\
& D_{\boldsymbol{n}} u(\boldsymbol{x})=\mathrm{v}(\boldsymbol{x})=\widehat{\mathrm{v}}(\boldsymbol{x}), \quad x \in \Gamma_{\mathrm{v}},
\end{aligned}
$$

where $\dot{\boldsymbol{x}} \in \Omega$ and $x \in \Gamma=\Gamma_{u} \cup \Gamma_{\mathrm{v}}$ are the current points belonging to the domain $\Omega$ and the boundary $\Gamma$, respectively, and $\widehat{u}(\boldsymbol{x}), \widehat{\mathrm{v}}(\boldsymbol{x})$ are given functions.

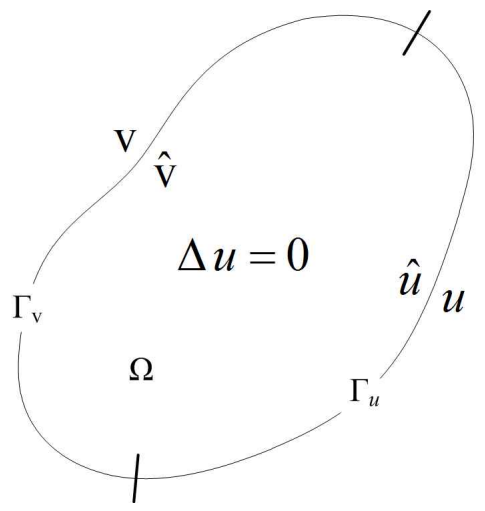

Fig. 1. Geometry of the general boundary problem.

The boundary problem described by Eqs. (1)-(3) is classically formulated.

The transformation of the classical formulation into variational formulations is done via the weighted residual method (WRM) [17]. Let the solution $u(\boldsymbol{x})$ be substituted by an approximate solution $\tilde{u}(\boldsymbol{x})$. Inserting $\tilde{u}(\boldsymbol{x})$ into Eqs. (1)-(3) generates residuals. The first step of WRM is multiplying Eq. (1) by any weighting function $W$ (briefly, by a weight) and integrating it over domain $\bar{\Omega}$, $\bar{\Omega}=\Omega \cup \Gamma$. Hence, the weighted integral equation is obtained. An idea of WRM consists in selection of appropriate weights in order to distribute the residuum on domain $\bar{\Omega}$ so the weighted integral equation should be equal to zero:

$$
\int_{\Omega} \Delta \tilde{u} W d \boldsymbol{x}=0, \quad \boldsymbol{x} \in \bar{\Omega} .
$$

Eq. (4) is the basic one of WRM and is called the weighted residual statement. At the same time, it is an original variational formulation of the boundary problem. Now, let one introduce boundary conditions into Eq. (4) via Green's second identity. After some calculations, one obtains original and inverse variational formulation, respectively, of the boundary problem described by Eqs. (1)-(3) with instilled boundary conditions, namely [18, 19]:

$$
\begin{aligned}
& \int_{\Omega} \Delta \tilde{u} W d \boldsymbol{x}+\int_{u} \tilde{u} D_{\boldsymbol{n}} W d \boldsymbol{x}-\int_{\mathrm{v}} \tilde{\mathrm{v}} W d \boldsymbol{x}= \\
& \int_{u} \widehat{u} D_{\boldsymbol{n}} W d \boldsymbol{x}-\int_{\mathrm{v}} \widehat{\mathrm{v}} W d \boldsymbol{x}, \\
& \int_{\Omega} \tilde{u} \Delta W d \boldsymbol{x}+\int_{u} \tilde{\mathrm{v}} W d \boldsymbol{x}-\int_{\mathrm{v}} \tilde{u} D_{\boldsymbol{n}} W d \boldsymbol{x}= \\
& \int_{u} \widehat{u} D_{\boldsymbol{n}} W d \boldsymbol{x}-\int_{\mathrm{v}} \widehat{\mathrm{v}} W d \boldsymbol{x} .
\end{aligned}
$$

Note that the right-hand sides in Eqs. (5) and (6) are the same.

\section{Forms of the approximate solution, bases, and weights}

The approximate solution $\tilde{u}$ is assumed in the form of series $\tilde{u}_{\Sigma}$,

$$
\tilde{u}=\tilde{u}_{\Sigma}=\sum_{\mathrm{v}} a_{\mathrm{v}} \phi_{\mathrm{v}}, \quad \mathrm{v}=1,2, \ldots n,
$$

where $a_{\mathrm{v}}$ are unknown coefficients and $\phi_{\mathrm{v}}$ are bases.

The bases $\phi_{\mathrm{v}}$ are made up with T-functions marked with $u^{\circ}(r), \boldsymbol{r}=\left|\boldsymbol{x}^{\prime}-\dot{\boldsymbol{x}}\right|$ or K-functions $u^{\bullet}\left(r_{\varepsilon}\right), \boldsymbol{r}_{\varepsilon}=$ $\left|\boldsymbol{x}_{\varepsilon}^{\prime}-\dot{\boldsymbol{x}}\right|$, Fig. 2, where the set $\left\{\boldsymbol{x}_{\varepsilon}^{\prime}\right\}$ describes distribution of sources on the fictitious boundary $\Gamma_{\varepsilon}$ and the set $\left\{\boldsymbol{x}^{\prime}\right\}$ describes distribution of influence points on the real boundary $\Gamma$; the influence points are equivalent to the source points if the bases constitute FS.

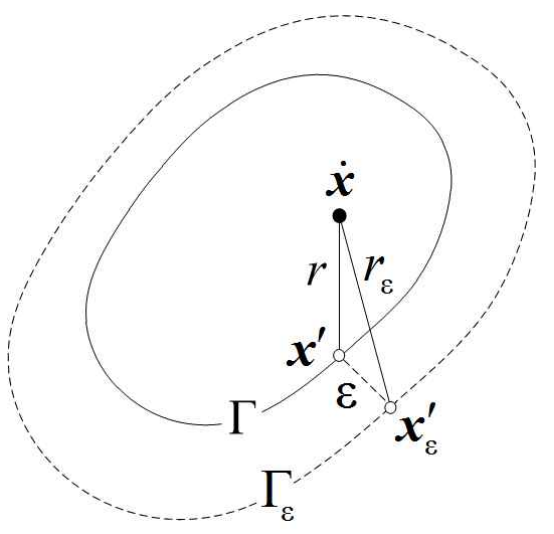

Fig. 2. Geometry of the boundary problem.

T-functions satisfy the homogeneous differential equation, i.e. $\Delta u^{\circ}(r)=0$, and they are not singular. Therefore $\mathrm{T}$-functions are defined separately both for exterior domain and for interior one. The set of $u^{\circ}(r)$, denoted by $\left\{u_{\mathrm{v}}^{\circ}(r)\right\}$, is described a priori. For interior domain $\Omega$ and two-dimensional problem, the T-functions have the form, $[6,7,18],\left\{1, r^{\mathrm{v}} \exp (i \mathrm{v} \varphi)\right\}, \mathrm{v}=1,2 \ldots$ or in explicit form $\left\{1, r \cos (\varphi), r \sin (\varphi), r^{2} \cos (2 \varphi), r^{2} \sin (2 \varphi), \ldots\right\}$.

The form of $u^{\bullet}\left(r_{\varepsilon}\right)$ is quite the same as FS for the non-homogeneous Laplace's equation and it solves $\Delta u^{\bullet}(r)=-\delta(r)$, where $\delta(r)$ is the Dirac delta function, $r=\left|\boldsymbol{x}^{\prime}-\dot{\boldsymbol{x}}\right|$. Following Kupradze's tracks, all sources $\left\{\boldsymbol{x}_{\varepsilon}^{\prime}\right\}$ are distributed outside $\bar{\Omega},\left\{\boldsymbol{x}_{\varepsilon}^{\prime}\right\} \notin \bar{\Omega}$ i.e. they are obtained moving $\boldsymbol{x}^{\prime}$ outside $\bar{\Omega}$. So, $u^{\bullet}\left(r_{\varepsilon}\right)$ is the solution to the homogeneous Laplace's equation, i.e. $\Delta u^{\bullet}\left(r_{\varepsilon}\right)=0$. For two-dimensional problem, one has $u^{\bullet}\left(r_{\varepsilon}\right)=1 /(2 \pi) \ln \left(1 / r_{\varepsilon}\right)$. The set of $\left\{u_{\mathrm{v}}^{\bullet}\left(r_{\varepsilon}\right)\right\}$ is derived from the formula $u_{\mathrm{v}+1}^{\bullet}\left(r_{\varepsilon}\right)=D_{\boldsymbol{n}} u_{\mathrm{v}}^{\bullet}\left(r_{\varepsilon}\right)$, where $u_{1}^{\bullet}\left(r_{\varepsilon}\right)=u^{\bullet}\left(r_{\varepsilon}\right), \boldsymbol{n}$ is the unit outward normal to $\Gamma$ at $\boldsymbol{x}^{\prime}$. As a result, the $u^{\bullet}\left(r_{\varepsilon}\right)$ is not singular, if $\dot{\boldsymbol{x}} \rightarrow \boldsymbol{x}$, $\{\boldsymbol{x}, \dot{\boldsymbol{x}}\} \in \Omega$ or $\boldsymbol{x}^{\prime} \rightarrow \boldsymbol{x},\left\{\boldsymbol{x}, \boldsymbol{x}^{\prime}\right\} \in \Gamma$.

It is worth to note that when the distance from the source increases, the K-functions are converge to T-functions. However, the methods with K-functions are much less stable than the methods with T-functions [8]. 


\section{Numerical methods}

Four methods are derived. In order to clearly distinguish differences between them, the symbols are used as proposed in [18], instead of descriptions. Each method can be described by a four-letter code. The first letter denotes the kind of the variational formulation from which the method is derived: $\mathrm{O}-[\mathrm{O} \mid$ riginal and $\mathrm{I}-$ [I]nverse. The second component of the code, after the hyphen, are two letters separated by semicolon. The first means that the solution is assumed to be a $\mathrm{S}$ - [S]eries expansion. The latter describes the bases; here the bases constitute T-functions and K-functions. The third component, again after the hyphen, is a fourth letter. It corresponds to the form of the weights; here they are made also by T-functions and K-functions.

Hereafter, the following nonsingular two groups of methods are considered: $\mathrm{O}-\mathrm{S} ; \mathrm{T}-\mathrm{T}, \mathrm{O}-\mathrm{S} ; \mathrm{K}-\mathrm{K}$ and $\mathrm{I}-\mathrm{S} ; \mathrm{T}-\mathrm{T}, \mathrm{I}-\mathrm{S} ; \mathrm{K}-\mathrm{K}$. The first group of methods is based on Eq. (5) while the second one on Eq. (6). All methods are the Galerkin versions, so it is justified to explore them together, and to provide their comparisons.

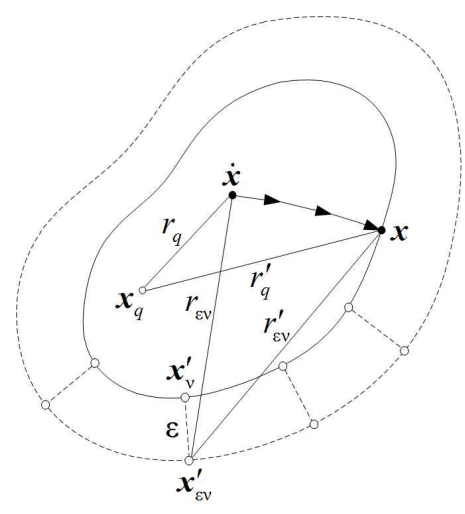

Fig. 3. Geometrical symbols of the bases.

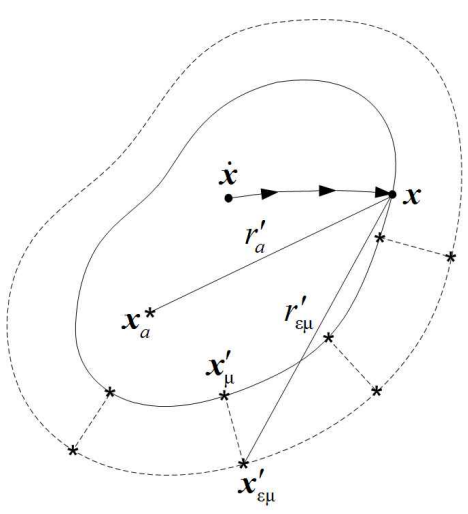

Fig. 4. Geometrical symbols of the weights.

In all TMs, the sources or influence points are denoted identically by open circles "o", the weights points are marked by asterisks "**", but the calculated points are marked by solid circles "•", Figs. 3 and 4 .
4.1. The first method of O-formulation; $O-S ; T-T$

In this case, the solution has the form

$$
\tilde{u}(r)=\sum_{\mathrm{v}} a_{\mathrm{v}} u_{\mathrm{v}}^{\circ} \text {. }
$$

Unknown coefficients $a_{\mathrm{v}}$, interpreted as intensity of the influence points, need to be calculated. First of all, the $u_{\mathrm{v}}^{\circ}$ can take one of the two forms, Fig. 3:

- $u_{\mathrm{v}}^{\circ}=u^{\circ}\left(r_{\mathrm{v}}\right), r_{\mathrm{v}}=\left|\boldsymbol{x}_{\mathrm{v}}-\dot{\boldsymbol{x}}\right|,\left\{\boldsymbol{x}_{\mathrm{v}}\right\}$ - the set of the influence points,

- $u_{\mathrm{v}}^{\circ}=u_{\mathrm{v}}^{\circ}\left(r_{q}\right), r_{q}=\left|\boldsymbol{x}_{q}-\dot{\boldsymbol{x}}\right|, \boldsymbol{x}_{q}$ - one influence point.

Both $\left\{\boldsymbol{x}_{\mathrm{v}}\right\}$ and $\boldsymbol{x}_{q}$ may be distributed arbitrarily and not necessarily on $\Gamma$. Below, the second version is considered and the $\boldsymbol{x}_{q}$ point is placed in the domain $\Omega$.

The set of weights is given by $W=\left\{u_{\mu}^{\circ}\right\}$. Note that $u_{\mu}^{\circ}$ may be also interpreted in two ways, Fig. 4:

- $u_{\mu}^{\circ}=u^{\circ}\left(r_{\mu}\right), r_{\mu}=\left|\boldsymbol{x}_{\mu}-\dot{\boldsymbol{x}}\right|$ and the set of $\left\{\boldsymbol{x}_{\mu}\right\}$ may be distributed arbitrarily,

- $u_{\mu}^{\circ}=u_{\mu}^{\circ}\left(r_{a}\right), r_{a}=\left|\boldsymbol{x}_{a}-\dot{\boldsymbol{x}}\right|$ and one point $\boldsymbol{x}_{a}$ is an arbitrary one and it may be placed anywhere.

Note that when this case of weight is considered, the points $\left\{\boldsymbol{x}_{\mu}\right\}$ or $\boldsymbol{x}_{a}$ may not be on $\Gamma$. Here the second variant is selected and $\boldsymbol{x}_{a}$ is chosen in domain $\Omega$.

Because of $\Delta u_{\mathrm{v}}^{\circ}=0$ and moving $\dot{\boldsymbol{x}} \in \Omega$ to $\boldsymbol{x} \in \Gamma$, Eq. (5) reduces to

$$
\begin{aligned}
& \sum_{\mathrm{v}} a_{\mathrm{v}}\left(\int_{u} u_{\mathrm{v}}^{\circ}\left(r_{q}^{\prime}\right) D_{\boldsymbol{n}} u_{\mu}^{\circ}\left(r_{a}^{\prime}\right) d \boldsymbol{x}\right. \\
& \left.-\int_{\mathrm{v}} D_{\boldsymbol{n}} u_{\mathrm{v}}^{\circ}\left(r_{q}^{\prime}\right) u_{\mu}^{\circ}\left(r_{a}^{\prime}\right) d \boldsymbol{x}\right)=\int_{u} \widehat{u}(\boldsymbol{x}) D_{\boldsymbol{n}} u_{\mu}^{\circ}\left(r_{a}^{\prime}\right) d \boldsymbol{x} \\
& -\int_{\mathrm{v}} \widehat{\mathrm{v}}(\boldsymbol{x}) u_{\mu}^{\circ}\left(r_{a}^{\prime}\right) d \boldsymbol{x},
\end{aligned}
$$

where $r_{q}^{\prime}=\left|\boldsymbol{x}_{q}-\boldsymbol{x}\right|, r_{a}^{\prime}=\left|\boldsymbol{x}_{a}-\boldsymbol{x}\right|,\left\{\boldsymbol{x}_{a}, \boldsymbol{x}_{q}\right\} \in \Omega$, $\{\boldsymbol{x}\} \in \Gamma$.

After some calculations, see e.g. [15, 20], this leads to the set of algebraic equations which can be written down in the matrix form

$$
A a=b,
$$

where $\boldsymbol{A}$ is the main matrix of the appropriate integrals, $\boldsymbol{a}$ is the vector of unknowns $\left\{a_{\mathrm{v}}\right\}$, and $\boldsymbol{b}$ is the vector of the known coefficients computed as the integrals from the known boundary conditions $\widehat{u}(\boldsymbol{x})$ and $\widehat{\mathrm{v}}(\boldsymbol{x})$. The BIE in the rest of the methods can be written down in the form of Eq. (10).

It is worth to note that the main matrix $\boldsymbol{A}$ in Galerkin versions is symmetric [11]. So its accuracy and computational efficiency are higher than those in the other formulations [21-23].

\subsection{The second method of O-formulation; $O-S ; K-K$}

The method has the solution in the form

$$
\tilde{u}(r)=\sum_{\mathrm{v}} a_{\mathrm{v}} u_{\varepsilon \mathrm{v}}^{\bullet} \text {. }
$$

Unknown coefficients $a_{\mathrm{v}}$, also interpreted as intensities of the sources, should be calculated. Two sets of bases can be generated, Fig. 3: 
- $u_{\varepsilon \mathrm{v}}^{\bullet}=u^{\bullet}\left(r_{\varepsilon \mathrm{v}}\right), r_{\varepsilon \mathrm{v}}=\left|\boldsymbol{x}_{\varepsilon \mathrm{v}}^{\prime}-\dot{\boldsymbol{x}}\right|$ and the set of $\left\{\boldsymbol{x}_{\varepsilon \mathrm{V}}^{\prime}\right\} \in \Gamma_{\varepsilon}$

- $u_{\varepsilon \mathrm{v}}^{\bullet}=u_{\mathrm{v}}^{\bullet}\left(r_{\varepsilon q}\right), r_{\varepsilon q}=\left|\boldsymbol{x}_{\varepsilon q}-\dot{\boldsymbol{x}}\right|$ and one point $\boldsymbol{x}_{\varepsilon q}$ ought to be placed anywhere but outside $\bar{\Omega}$.

Here, the first variant is chosen. It means that instead of sources of high orders, a finite number of sources of the first order is applied. Thus the set $\left\{u^{\bullet}\left(r_{\varepsilon \mathrm{V}}\right)\right\}$ is used instead of $\left\{u_{\mathrm{v}}^{\bullet}\left(r_{\varepsilon q}\right)\right\}$; and consequently, $\tilde{\mathrm{v}}(\boldsymbol{x})=D_{\boldsymbol{n}} u^{\bullet}=$ $\sum_{\mathrm{v}} D_{\boldsymbol{n}} u^{\bullet}\left(r_{\varepsilon \mathrm{v}}\right)$.

The set of weights is also given by $\mathrm{K}$-functions, i.e. $W=\left\{u_{\varepsilon \mu}^{\bullet}\right\}$. Quite similar as above $u_{\varepsilon \mu}^{\bullet}$, may be also interpreted in two ways, Fig. 4:

$$
\begin{aligned}
& \text { - } u_{\varepsilon \mu}^{\bullet}=u^{\bullet}\left(r_{\varepsilon \mu}\right), r_{\varepsilon \mu}=\left|\boldsymbol{x}_{\varepsilon \mu}^{\prime}-\dot{\boldsymbol{x}}\right|, \\
& \text { - } u_{\varepsilon \mu}^{\bullet}=u_{\mu}^{\bullet}\left(r_{\varepsilon a}\right), r_{\varepsilon a}=\left|\boldsymbol{x}_{\varepsilon a}-\dot{\boldsymbol{x}}\right| .
\end{aligned}
$$

In this case the first variant is also chosen. Note that when this type of weights is chosen, the points $\left\{\boldsymbol{x}_{\varepsilon \mu}^{\prime}\right\}$ or $\boldsymbol{x}_{\varepsilon a}$ may be placed anywhere and not necessarily on $\Gamma_{\varepsilon}$, but here it is assumed that $\left\{\boldsymbol{x}_{\varepsilon \mu}^{\prime}\right\}$ are distributed on $\Gamma_{\varepsilon}$.

Since $\Delta u^{\bullet}\left(r_{\varepsilon \mathrm{v}}\right)=0$, the integral over the domain $\Omega$ in Eq. (5) is equal to zero. Shifting $\dot{\boldsymbol{x}} \in \Omega$ to $\boldsymbol{x} \in \Gamma$, Eq. (5) can be rewritten as BIE,

$$
\begin{aligned}
& \sum_{\mathrm{v}} a_{\mathrm{v}}\left(\int_{u} u^{\bullet}\left(r_{\varepsilon \mathrm{v}}^{\prime}\right) D_{\boldsymbol{n}} u^{\bullet}\left(r_{\varepsilon \mu}^{\prime}\right) d \boldsymbol{x}\right. \\
& \left.\quad-\int_{\mathrm{v}} D_{\boldsymbol{n}} u^{\bullet}\left(r_{\varepsilon \mathrm{v}}^{\prime}\right) u^{\bullet}\left(r_{\varepsilon \mu}^{\prime}\right) d \boldsymbol{x}\right)= \\
& \int_{u} \widehat{u}(\boldsymbol{x}) D_{\boldsymbol{n}} u^{\bullet}\left(r_{\varepsilon \mu}^{\prime}\right) d \boldsymbol{x}-\int_{\mathrm{v}} \widehat{\mathrm{v}}(\boldsymbol{x}) u^{\bullet}\left(r_{\varepsilon \mu}^{\prime}\right) d \boldsymbol{x},
\end{aligned}
$$

where for the sake of brevity hereafter, Figs. 3, 4,

$$
\begin{aligned}
& r_{\varepsilon \mathrm{v}}^{\prime}=\left|\boldsymbol{x}_{\varepsilon \mathrm{v}}^{\prime}-\boldsymbol{x}\right|, r_{\varepsilon \mu}^{\prime}=\left|\boldsymbol{x}_{\varepsilon \mu}^{\prime}-\boldsymbol{x}\right|, \\
& \left\{\boldsymbol{x}_{\varepsilon \mathrm{v}}^{\prime}, \boldsymbol{x}_{\varepsilon \mu}^{\prime}\right\} \in \Gamma_{\varepsilon},\{\boldsymbol{x}\} \in \Gamma .
\end{aligned}
$$

The next two methods that belong to I-formulation are based on Eq. (6). The methods are derived under the same assumptions as the methods of O-formulations so these assumptions are not repeated. That is why they are considered jointly in the next subsection.

\subsection{Methods of I-formulation}

The next method is $\mathrm{I}-\mathrm{S} ; \mathrm{T}-\mathrm{T}$ (compare to $\mathrm{O}-\mathrm{S} ; \mathrm{T}-\mathrm{T}$ ). Therefore Eq. (6) leads to the following BIE:

$$
\begin{aligned}
& \sum_{\mathrm{v}} a_{\mathrm{v}}\left(\int_{u} D_{\boldsymbol{n}} u_{\mathrm{v}}^{\circ}\left(r_{q}^{\prime}\right) u_{\mu}^{\circ}\left(r_{a}^{\prime}\right) d \boldsymbol{x}\right. \\
& \left.-\int_{\mathrm{v}} u_{\mathrm{v}}^{\circ}\left(r_{q}^{\prime}\right) D_{\boldsymbol{n}} u_{\mu}^{\circ}\left(r_{a}^{\prime}\right) d \boldsymbol{x}\right)= \\
& \int_{u} \widehat{u}(\boldsymbol{x}) D_{\boldsymbol{n}} u_{\mu}^{\circ}\left(r_{a}^{\prime}\right) d \boldsymbol{x}-\int_{\mathrm{v}} \widehat{\mathrm{v}}(\boldsymbol{x}) u_{\mu}^{\circ}\left(r_{a}^{\prime}\right) d \boldsymbol{x} .
\end{aligned}
$$

The last method is $\mathrm{I}-\mathrm{S} ; \mathrm{K}-\mathrm{K}$; compare to $\mathrm{O}-\mathrm{S} ; \mathrm{K}-\mathrm{K}$. So, the BIE derived from Eq. (6) comes down to

$$
\begin{gathered}
\sum_{\mathrm{v}} a_{\mathrm{v}}\left(\int_{u} D_{\boldsymbol{n}} u^{\bullet}\left(r_{\varepsilon \mathrm{v}}^{\prime}\right) u^{\bullet}\left(r_{\varepsilon \mu}^{\prime}\right) d \boldsymbol{x}\right. \\
\left.-\int_{\mathrm{v}} u^{\bullet}\left(r_{\varepsilon \mathrm{v}}^{\prime}\right) D_{\boldsymbol{n}} u^{\bullet}\left(r_{\varepsilon \mu}^{\prime}\right) d \boldsymbol{x}\right)=
\end{gathered}
$$

$$
\int_{u} \widehat{u}(x) D_{\boldsymbol{n}} u^{\bullet}\left(r_{\varepsilon \mu}^{\prime}\right) d \boldsymbol{x}-\int_{\mathrm{v}} \widehat{\mathrm{v}}(x) u^{\bullet}\left(r_{\varepsilon \mu}^{\prime}\right) d \boldsymbol{x} .
$$

\section{Numerical calculations, results}

\subsection{Research object}

There are no limitations as for the boundary problem, i.e. its geometry and boundary conditions. But it is recommended to choose such a problem which has an analytical solution. The Motz's problem is a benchmark of singularity problems and it is selected as a prototype for verifying the above-described methods. This problem solves the Laplace's equation $\Delta u(r)=0$ on the rectangle $x \in(-1,1)$ and $y \in(0,1)$ with Neumann-Dirichlet boundary conditions, Fig. 5 ,

$$
\begin{aligned}
& u=\widehat{u}=0, \quad x \in(-1,0) \cap y=0, \\
& u=\widehat{u}=100, \quad x=1 \cap y \in(0,1), \\
& D_{\boldsymbol{n}} u=\widehat{\mathrm{v}}=0, \quad \text { elsewhere. }
\end{aligned}
$$

The solution of the Motz's problem is given in [10, 24],

$$
\begin{aligned}
& u(r, \varphi)=\sum_{\mathrm{v}} c_{\mathrm{v}} r^{\mathrm{v}+1 / 2} \cos (\mathrm{v}+1 / 2) \varphi, \\
& \mathrm{v}=1,2, \ldots, n,
\end{aligned}
$$

where $c_{\mathrm{v}}$ are expansion coefficients and $(r, \varphi)$ are polar coordinates with the origin at $(0,0)$.

The solution Eq. (17) satisfies the Laplace's equation and boundary condition for $x \in(-1,1)$ and $y=0$ automatically. The coefficients $c_{\mathrm{v}}$ are sought by satisfying the other boundary conditions.

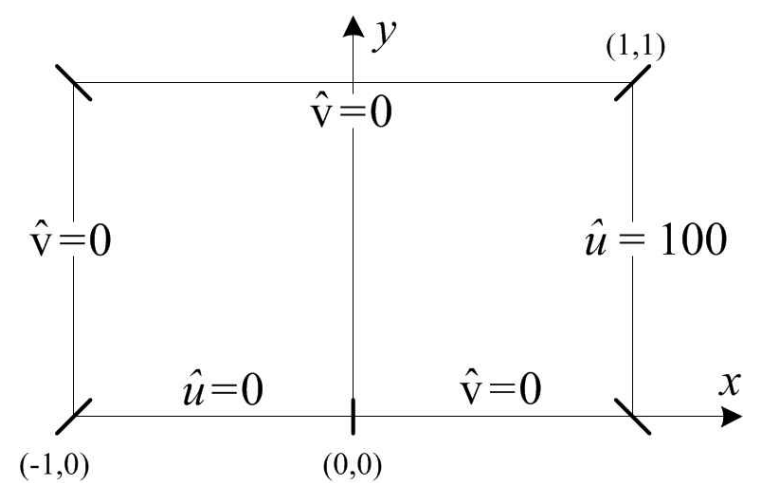

Fig. 5. The Motz's problem.

\subsection{Results}

In this section, the numerical experiments for the Motz's problem are discussed. The errors of TMs are measured via the $[\mathrm{R}]$ oot $[\mathrm{M}]$ ean $[\mathrm{S}]$ quare distance,

$$
E_{\mathrm{RMS}}=\left(\sum_{i}\left(\tilde{u}_{i}-u_{i}\right)^{2} / n_{i}\right)^{1 / 2},
$$

where $n_{i}$ is the number of the calculated points $\boldsymbol{x}_{i} \in \Omega$.

In order to compare the methods presented in the preceding section, some numerical results are quoted. In all 


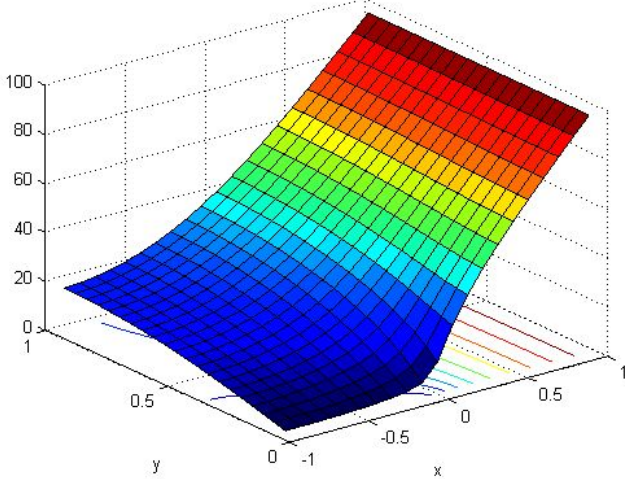

Fig. 6. Exact solution of the Motz's problem.
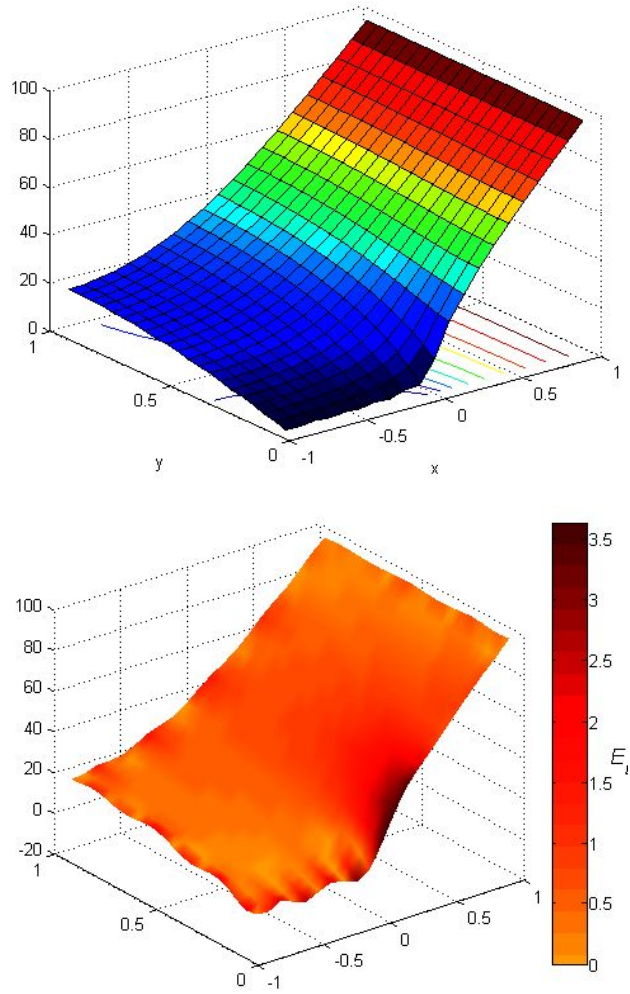

Fig. 7. Approximate solution and the errors of $\mathrm{O}-\mathrm{S} ; \mathrm{T}-$ $\mathrm{T} / \mathrm{I}-\mathrm{S}$; T-T: (a) $\tilde{u}, E_{\mathrm{RSM}}=0.7287$, (b) false colored by error $E_{L}=|u-\tilde{u}|$.

numerical experiments, the following parameters are assumed: $\boldsymbol{x}_{q}=\boldsymbol{x}_{a}=(0,0.5)$, hence the point is placed in the middle of the domain $\Omega$, the source points are moved away from the real boundary $\Gamma$ to the artificial one $\Gamma_{\varepsilon}$ by $\varepsilon=\varepsilon_{\mathrm{v}}=3$, Fig. 3, whereas the weight points by $\varepsilon=\varepsilon_{\mu}=3$, Fig. 4 . Both distances are based on numerical experiments but they are not confirmed with theoretical considerations; it seems to be a very difficult task. The number of source points $\boldsymbol{x}_{\varepsilon \mathrm{v}}^{\prime}$ and the number of weight points are equal, $n=m=40$.

The exact solution is depicted in Fig. 6. This is the reference result. The solutions obtained by each of the
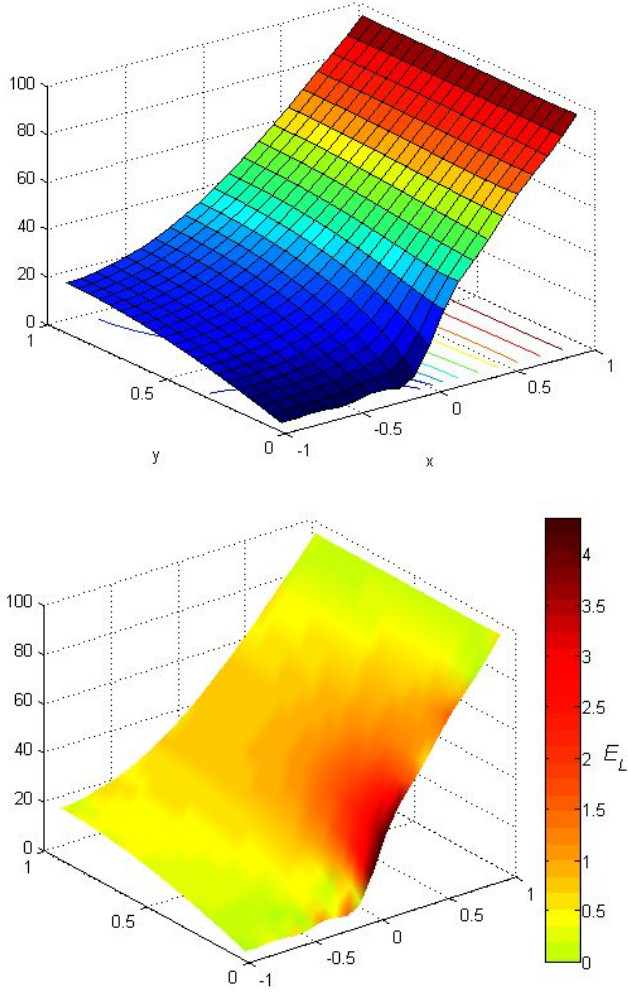

Fig. 8. Approximate solution and the errors of $\mathrm{O}-\mathrm{S}$; $\mathrm{K}-\mathrm{K} / \mathrm{I}-\mathrm{S} ; \mathrm{K}-\mathrm{K}$ : (a) $\tilde{u}, E_{\mathrm{RMS}}=0.7922$, (b) false colored by error $E_{L}=|u-\tilde{u}|$.

methods are presented in Figs. 7 and 8. Since the results are the same within each pair, they both are presented in common figures.

\section{Concluding remarks}

As can be seen, the TM method may be considered, for instance as a generalization of the method of separation of variables. In classical one (Fourier method), the basic set of eigenfunctions has to be particular to a differential equation, to a specific geometry, and boundary conditions. The TM weakens these requirements, namely, it requires that the set of bases satisfies the homogeneous governing equation. However, when calculating a set of unknown coefficients, the boundary conditions are satisfied in some manner on the given geometry. This is a first advantage from the point of view of engineering applications.

As it has been mentioned in the introduction, TMs belong to the boundary-type methods. The advantages include a simple treatment of physical and geometrical singularities. Additionally, the data preparation requires only a geometrical definition of the boundaries and not of the mesh within. This is the second benefit for engineers.

In the paper, two groups of the Galerkin version methods containing nonsingular T-functions and K-functions are presented: the former based on the original variational formulation of the problem, the latter — on the 
inverse one. In the Galerkin version, the base, as well as the weight, take the same form, of either T-functions or K-functions. Since T-functions and K-functions are nonsingular, therefore the methods are also nonsingular. All the methods are applied for solving numerically 2-D Motz's problem. Based on this example, the efficiency and accuracy of separate methods, expressed by means of the root mean square distance between the analytical and approximate solutions, are calculated. The following conclusions can be drawn from the above-presented considerations:

1. It is proved that, under certain conditions, the group of methods in Galerkin versions of O-formulation is related to the group of methods of I-formulation, i.e. O-S;T-T/I-S;T-T and O-S;K-K/I-S;K-K. Furthermore, the main matrices have the property of symmetry.

2. Taking into account the efficiency and accuracy, the methods $\mathrm{O}-\mathrm{S} ; \mathrm{T}-\mathrm{T} / \mathrm{I}-\mathrm{S} ; \mathrm{T}-\mathrm{T}$ give the best results, so $\mathrm{O}-\mathrm{S} ; \mathrm{T}-\mathrm{T} / \mathrm{I}-\mathrm{S} ; \mathrm{T}-\mathrm{T}$ are superior to $\mathrm{O}-\mathrm{S} ; \mathrm{K}-\mathrm{K} / \mathrm{I}-\mathrm{S} ; \mathrm{K}-\mathrm{K}$.

3. Since all methods may serve as efficient numerical methods for solving engineering problems, in the future work the authors intend to employ them for solving the magnetic and electric field distributions of the transformer.

\section{Acknowledgments}

Numerical experiments were conducted with the use of MATLAB software purchased under the Project No. UDA-RPPK.01.03.00-18-003/10-00 "Construction, expansion and modernization of the scientific-research base at Rzeszów University of Technology" co-financed by the European Union from the European Regional Development Fund within Regional Operational Programme for Podkarpackie Region for the years 2007-2013, I. Competitive and innovative economy, 1.3 Regional innovation system.

\section{References}

[1] E. Trefftz, Proc. 2nd Int. Cong. Appl. Mech., Zurich 1926, p. 131.

[2] P.K. Banerjee, R. Butterfield, Boundary Element Methods in Engineering Science, McGraw-Hill Book, London 1981, e-book 2007.

[3] L.C. Wrobel, The Boundary Element Method, Vol. 1, Appliacation in Thermo-Fluids and Acoustics, John Wiley \& Sons, Chichester 2002.
[4] R.D. Ciskowski, C.A. Brebbia, Boundary element methods in acoustics, Computational Mechanics Publications, Southampton 1991.

[5] I. Herrera, Boundary methods; An algebraic theory, Pitman Publishing, Boston 1984.

[6] A.P. Zieliński, Adv. Eng. Softw. 24, 147 (1995).

[7] I. Herrera, Numer. Meth. Partial Differ. 16, 561 (2000).

[8] Z-C. Li, L-J. Young, H-T. Huang, Y-P. Liu, A.H.-D. Cheng, EABE 34, 248 (2010).

[9] A. H-D. Cheng, T-T. Lu, Trefftz and collocation methods, WIT Press, Southampton 2006.

[10] Zi-Cai Li, T-T. Lu, H-T. Hu, A.H.-D. Cheng, Trefftz and collocation methods, WIT Press, Southampton 2008 .

[11] E. Kita, N. Kamiya, Adv. Eng. Softw. 24, 3 (1995).

[12] R. Schaback in Adaptive numerical solution of MFS systems, Eds. C.S. Chen, A. Karegeorghis, Y.S. Smyrlis, Dynamic Publishers, Atlanta 2008, p. 1.

[13] J.T. Chen, C.S. Wu, Y.T. Lee, K.H. Chen, Comput. Math. Appl. 53, 851 (2007).

[14] P. Gamallo, R.J. Astley, Int. J. Numer. Meth. Engng. 71, 406 (2007).

[15] A. Brański, M. Borkowski, D. Borkowska, EABE 36 , 505 (2012).

[16] Kok Hwa Yu, A. Halim Kadarman, H. Djojodihardio, EABE 34, 884 (2010).

[17] R. Henda, Advanced process simulation, Method of Weighted Residuals (lecture), Lund, Sweden 2006.

[18] A. Brański, Numerical methods for solving boundary problems, survey and classification, Publishing House Rzeszow University of Technology, Rzeszow 2013 (in Polish).

[19] C.A. Brebbia, J.C.F. Telles, L.C. Wrobel, Boundary Element Techniques, Springer-Verlag, Berlin, New York 1984.

[20] D.J. Cartwright, Underlying principles of the boundary element method, WIT Press, Southampton 2001.

[21] Y.K. Cheung, W.G. Jin, O.C. Zienkiewicz, Comm. Appl. Num. Meth. 5, 159 (1989).

[22] W.G. Jin, Y.K. Cheung, O.C. Zienkiewicz, Int. J. Numer. Meth. Engng 30, 1147 (1990).

[23] Y.K. Cheung, W.G. Jin, O.C. Zienkiewicz, Int. J. Numer. Meth. Engng 32, 63 (1991).

[24] Zi-Cai Li, T-T. Lu, H-T. Huang, A.H.-D. Cheng, Numer. Meth. Part. D. E. 23, 93 (2007). 